\title{
Case Report: Positive outcome of medical leeches (hirudotherapy) for venous congestion
}

\author{
P Brzezinski ${ }^{1}$, C Solovan ${ }^{2}$, A Chiriac $^{3}$, L Foia $^{4}$ \\ 1. Head of Department of Dermatology, 6th Military Support Unit, \\ Ustka, Poland \\ 2. Head of Department of Dermatology, University of Medicine V \\ Babes, Timisoara, Romania \\ 3. Head of Department of Dermato-Physiology, Apollonia University \\ Iasi, Strada Muzicii nr 2, Iasi-700399, Romania \\ 4. Head of Department of Dermatology, Grigore T. Popa University of \\ Medicine and Pharmacy, Strada Universitãtii 16, Iasi, Romania \\ Corresponding author: Piotr Brzezinski, Email-brzezoo@wp.pl
}

\section{Case Presentantion}

A 65 year Caucasian woman was seen for numerous, ovalto-round, well-defined 1.5 to $3.5 \mathrm{~cm}$ erythematous plaquelesions, with central umbilication (central black eschar), intensely itching, distributed on both inferior limbs, (Figures $1,2,3$ ). She admitted a history of leech therapy (3 sessions daily) 5 days prior to consultation, for chronic venous insufficiency under the supervision of a general practitioner. The patient had no pain or any other symptom, no fever, malaise. There were no palpable lymph nodes. Moreover, no history of trauma or insect bites could be recorded. She has been treated for several years for chronic superficial venous insufficiency with compression therapy (stockings) and micronized diosmin orally. She had no past or present trophic ulcers, just slight bilateral edema. A routine blood test was within normal limits. Echo Doppler evaluation confirmed chronic venous disease (CVD) class II and excluded venous thrombosis. Based on clinical examination, in the presence of normal investigations, we were in the position of deciding if it was a common evolution of hirudotherapy or an allergic contact dermatitis caused by the leech bite. The patient was treated with oral cefuroxime $1 \mathrm{~g}$ daily and antihistamines for 7 days, associated to topical steroids cream class II for 2 weeks, resulting in almost complete recovery. The patient was subsequently lost to follow-up.

Figure 1: Multiple lesions on the left leg

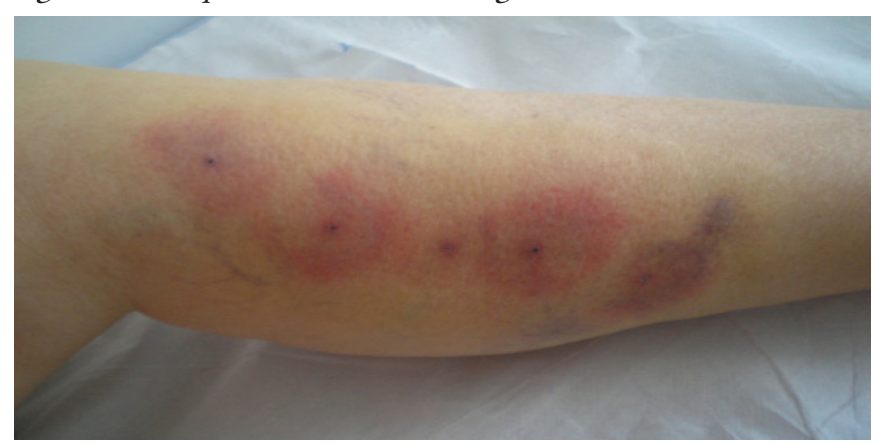

Figure 2: Round-oval erythematous plaques centered by small ulceration covered by black and adherent eschar on the inner surface of the right thigh

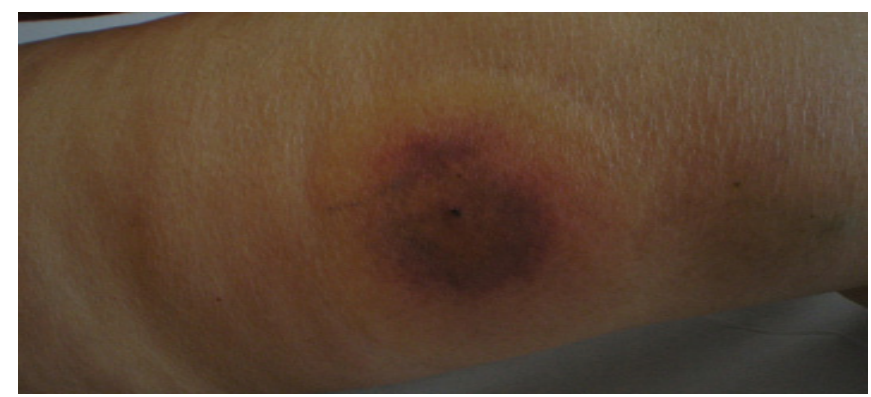

Figure 3: Close view of the lesion

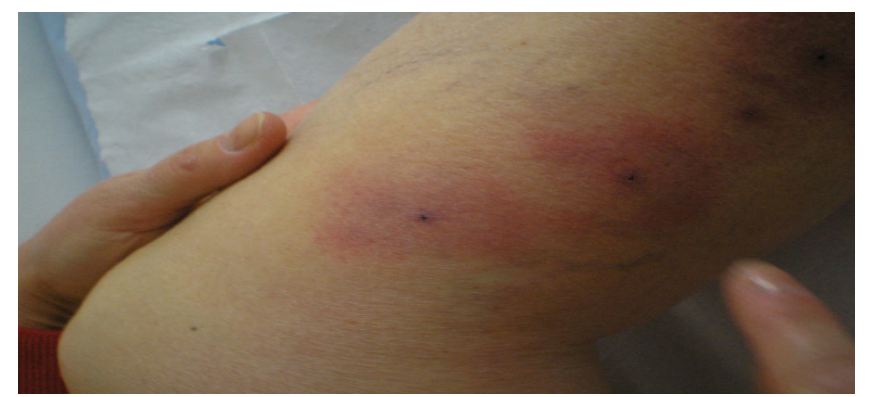

Figure 4: Hirudo medicinalis

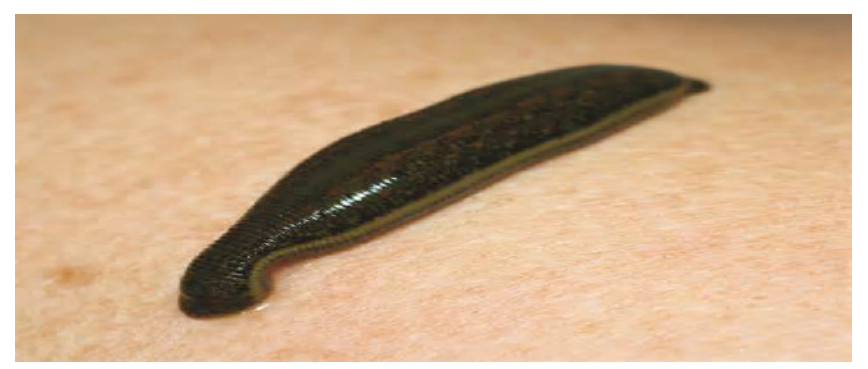

\section{Discussion}

The medicinal use of leeches goes far away back in the history. Galen (130-201 AD) used the technique to remove blood and provide health to patients ${ }^{2}$ but, the first use of leeches for medical purposes appears on the wall of an Egyptian tomb (1567-1308 BC) ${ }^{1}$. During centuries it became a popular remedy for many medical problems, but declined after the mid-nineteenth century due to the development of new medicine. In 2004, this treatment procedure received the approval of the Food and Drug Administration (FDA) in the USA ${ }^{2}$.

Leeches belong to the Phylum Annelids; they live in water (lakes, streams, seas, pools) or land in tropical areas. European leech Hirudo medicinalis is used in medicine and gave the name of hirudotherapy to the medical procedure, (Figure 4). When it is applied to skin Hirudo medicinalis sucks blood directly through the external mammalian skin. At the moment of biting, saliva of the Hirudo medicinalis releases into the skin substances that induce local anesthesia and vasodilatation (with enhanced blood flow); fibrinase 
and anticoagulant (hirudin) to prevent clotting ${ }^{3}$. Depending on the size of the leech, a large quantity of blood can be ingested.Side effects, accidents, medical problems have been described during or after using this method. Psychological impact can be significant and might push cessation of the procedure, with or without anxiolytic medication.Continued and uncontrolled leech use can induce bleeding and anaemia. Allergic local reactions and anaphylaxis have been reported, induced by substances contained in saliva of leeches ${ }^{4}$. Local infections with Aeromonas spp are by far the mostdangerous complications, ranging from superficial to deep infections (cellulitis, subcutaneous abscess) $^{5}$ and even septicemia8. High Resistance to first-generation Cephalosporin, Penicillin, Tetracycline, Augmentin widely used in daily practice, have been reported recently with the recommendation of prescribing fluoroquinolones ${ }^{6}$. Moreover, the multiple uses of the same leeches from one patient to another induces high risk of hepatitis and HIV transmission. The leech therapy can be used effectively for the management of various disorders. Rasi et all described 64-year-old Iranian man who presented with numerous asymptomatic multilobular oval-to-round cystic lesions ${ }^{3}$. The patient used leech therapy with positive effect. Zaidi descibes using leech therapy in a 60-year-old woman suffering from diabetic foot (she was facing the prospect of imminent amputation) 7 . Wound dressing was done with unripe papaya as it has a very good role in clearing necrotising area and hirudotherapy was also used in poorly healing wounds. The pain score decreased to $0-10 \mathrm{~mm}$ on a $100 \mathrm{~mm}$ visual analogue scale within 20 days and no further pain relieving medication was required. Over a time interval of nearly 3.5 months, necrotic areas disappeared and the wound was completely healed. Authors from India described a patient with nevus of Ota where leech was applied upon the lesion for five times spanned in a period of 2 months ${ }^{8}$. A substantial reduction in color of the nevus was reported following the completion of the therapy. An interesting case was presented by TarazJamshidi et $\mathrm{al}^{9}$. The authors presented a case of leech therapy after near total amputation of the fingers. A 25-year-old patient was admitted following a sawing injury with crashed bundles of the third, fourth and fifth fingers. Microvascular surgery was not performed because of crush injury. The patient was treated using leech therapy. The result was satisfactory. The third and fourth fingers were salvaged.

\section{Present case}

Besides being rare for practical dermatological activity, leech therapy raises a few questions:

-Are skin lesions "normal" reactions to leech therapy or must they be regarded and treated as an allergic contact dermatitis with the recommendation of discontinuing the therapy?

-Could the lesions be the expresssion of a consumption coagulopathy induced by substances from saliva of leeches?

-Is hirudotherapy of any help in providing relief in patients with chronic superficial venous insufficiency class I, II in the absence of complications?

-How can be proved the value of the method by objective paraclinical investigations?

The leech therapy has unique features; the leech sucks venous blood and aids ulcer healing, and can therefore be used as an effective adjunct in the management of complicated varicose veins and venous congestion. Iranian authors showed that (in animal models) the process of wound healing was significantly faster in the group treated with leech therapy $(p<0.05)$ than in the group treated with the topical phenytoin ${ }^{10}$. It may be used for the restoration of normal heath through its prophylactic and palliative action. However, there is the need to develop standard procedures and scientific parameters so that the efficacy of leech therapy can be proved in a rational manner.

\section{References}

1. Whitaker IS, Rao J, Izadi D, Butler PE. Hirudomedicinalis: ancient origins of leeches, and trends in the use of medicinal leeches throughout history. Br J Oral Maxillofac Surg 2004; 42(2):133-137.

2. Mumcuoglu KY, Huberman L, Cohen R, Temper V, Adler A, Galun $\mathrm{R}$, et al. Elimination of symbiotic Aeromonas spp. from the intestinal tract of the medicinal leech, Hirudo medicinalis, using ciprofloxacin feeding. Clin Microbiol Infect 2010; 16(6):563-567.

3. Rasi A, Faghihi A, Jalali MA, Zamanian A, Ghaffarpour G. Leech therapy for epidermoid cysts and review of the literature. Adv Biomed Res 2014; 31(3):112.

4. Wells MD, Manktelow RT, Boyd JB, Bowen V. The medical leech: an old treatment revisited. Microsurgery 1993; 14(3):183-186.

5. Evans J, Lunnis PJ, Gaunt PN, Hanley DJ. A case of septicaemia due to Aeromonas hydrophila.Br J Plast Surg 1990; 43(3):371-372.

6. Bauters TG, Buyle FM, Verschraegen G, Vermis K, Vogelaers D, Claeys $\mathrm{G}$, et al. Infection risk related to the use of medicinal leeches. Pharm World Sci 2007; 29:122-125.

7. Zaidi SA. Unani treatment and leech therapy saved the diabetic foot of a patient from amputation. Int Wound J. 2014 May 8. doi: 10.1111/ iwj. 12285.

8. Rastogi S, Chaudhari P Pigment reduction in nevus of Ota following leech therapy. J Ayurveda Integr Med. 2014;5(2):125-128.

9. TarazJamshidi M, Bagheri F, Mirkazemi M, Amelfarzad S, Ashraf $\mathrm{H}$, Azami M, et al. Leech therapy in nearly total amputation of fingers without vascular repair: a case report. Iran Red Crescent Med J. 2014;16(5):e6897.

10. Darestani KD, Mirghazanfari SM, Moghaddam KG, Hejazi S. Leech therapy for linear incisional skin-wound healing in rats. J Acupunct Meridian Stud. 2014;7(4):194-201. 